\title{
Neue Bienenarten aus Süd-Amerika.
}

\section{Von Dr. H. Friese, Schwerin i. M.}

Seit einer Reihe von Jahren verdanke ich Herrn Adolf $\mathrm{Ducke}$ in Para ein umfangreiches Material an Bienen, das er auf seinen mannigfachen Reisen im Amazonas-Gebiet sammeln konnte und mir zur Sichtung und event. Benennung übergab. Soweit ich vermochte, kam ich seinem Wunsche nach, gab ihm die Namen der alten Autoren und fügte die Namen der vermutlich neuen Spezies bei. Allmählich wächst dies Material nun beträchtlich an, so dafs ich im folgenden die Beschreibung der n. sp. folgen lasse, um bei genügender Orientierung mit weiterem Materiale auch die Monographien der wichtigen Genera in Angriff $\mathrm{zu}$ nehmen.

Die wichtigen biologischen Daten wird Herr A. D u cke uns später in seinen besonderen Arbeiten vorlegen; zur leichteren Orientierung gebe ich seine Nummer den einzelnen Arten bei, um die Kontrolle zu erleichtern. Dafs ich auch weiteres Material von anderen Sammlern und aus anderen Gegenden mit einflechte, bedarf wohl keiner besonderen Rechtfertigung.

\section{Anthidium duckei n. sp. or $\sigma^{\text {P }}$.}

Eine kleine breite Art wie A. indescriptum D. T. (= cognatum Sm.) von Amazonas, aber Scutellum breit gelb gezeichnet und auch Segment 1-2.

․ Schwarz, kaum noch braun behaart, Kopf und Thorax grob und dicht runzlig - punktiert, matt; Labrum und Mandibel ganz schwarz, Clypeus jederseits mit kleinem, gelbem Fleck, innere Orbita unregelmälsig breit gelb, Fleck an der Antennenbasis gelb, und ein langer, gelber Streifen an der äufseren Orbita; Antenne schwarzbraun, Scapus und drittes Geifselglied unten rot. Mesonotum vorne jederseits mit gelber Linie, Calli h. hinten gelb befilzt, Scutellum gerundet, kaum vorragend, breit gelb bandiert, auch das Nebenschildchen; Area matt, sehr fein gerunzelt, mit einzelnen gröfseren Punkten, mit längsriefigem Basalstreifen. Abdomen fein punktiert, glänzend, Segment $1--6$ gelb bandiert auf der Scheibe, auf Segment 1-2 meist unterbrochen, auf dem ersten zu runden Seitenflecken reduziert, aber der ursprüngliche Bindenverlauf rotbraun markiert, sechstes braun gefranst. Ventralsegmente gelbbraun, Scopa gelblich. Beine schwarzbraun, goldgelb behaart, Calcar rot. Flügel gelblich getrübt, Adern und Tegulae schwarzbraun. - L. $7^{1 / 2}-8 \mathrm{~mm}$, Br. $3 \mathrm{~mm}$. 
$\sigma^{7}$ wie + , aber auch Mandibelbasis, Clypeusrand und Antennenschaft vorne gelb, Antennen lang, erreichen das Scutellum; Abdomen dunkler, Segment 1-2 oft schwarzbraun werdend, Segment 1 auch gelb bandiert, einfach gerundet, unbewehrt. Ventralsegmente lang weifs gefranst. Beine schwarzbraun. - L. $6^{1} / 2-7 \mathrm{~mm}$, Br. $2 \frac{1}{2} \mathrm{~mm}$.

$\sigma^{7}+$ zahlreich durch $\mathrm{D} \mathrm{u} \mathrm{ck}$ e erhalten, von Para im Dezember, Januar bis Februar fliegend, von Obidos (im Juli) und von Santarem (im August).

Amazonas (Brasil).

Anthidium portoin. sp. 주오.

Kleine breite Art wie $A$. duckei, aber im $q$ durch die gelben, verlängerten Mandibel auffallend, die an der Basis bedornt sind, auch Clypeus in Dorn auslaufend, $\sigma^{7}$ Kopf und Segment 7 unbewehrt.

ㅇ. Schwarz, gelb gezeichnet, kurz gelbbraun behaart, Kopf und Thorax dicht punktiert, fast matt, am Kopf stellenweise gerunzelt, gelb sind: Mandibel, Clypeusvorderhälfte, Nebengesicht bis zum Scheitel, Antennenschaft vorne, Doppelkiel zwischen den Antennen und der seitliche wie hintere Kopfrand breit; Clypeus breit, stumpf, mitten in kleinen Dorn verlängert, Labrum mit 2 grofsen, rundlichen Höckern, Mandibel sehr lang, sichelartig verlängert, mit 3 kleinen Zähnen am Ende, an der Basis mit kurzem, dickem, nach vorne gerichtetem Dorn bewehrt; Antenne schwarz, zwischen den Antennen mit gelbem, scharfem Doppelkiel. Mesonotum mit 2 breiten, gelben Längsstreifen, die vorne nach dem Seitenrande umbiegen und fast ein $\boldsymbol{U}$ bilden; Scutellum mit breitem, gelbem Hinterrande, Nebenschildchen gelb, Area an der Basis grob längsrunzlig, sonst glatt, glänzend, mit einzelnen Punkten. Abdomen fein und dicht punktiert, seitlich grober punktiert, Segment $1-2$ fast schwarz, $3-6$ mit breiter, gelber Binde. Ventralsegmente gelb bandiert, 6 ganz gelb, Scopa weifslich. Beine braun. Flügel gelblich, mit dunkler Spitze, Adern gelblich, Tegulae rotbraun. - L. $8-9 \mathrm{~mm}$, Br. $3^{1} / 2 \mathrm{~mm}$.

$\sigma^{7}$ wie $q$, aber Mandibel und Clypeus unbewehrt, gelb gezeichnet, Segment 7 ganzrandig, gelb und schwach ausgebuchtet, sonst unbewehrt, Ventralsegment $3-5$ eingedrückt, wie bei Stelis, lang weifs behaart. - L. $7-8 \mathrm{~mm}$, Br. $3 \mathrm{~mm}$.

q var. Kopf und Thorax fast ohne Gelb, Flügel mit breitem, schwarzbraunem Endrand. var. nigritulum n. var.

$\sigma^{7}$ 우 einzeln von Para durch $\mathrm{Ducke}$ erhalten (Nr. 140), am 6. I. und wieder am 30. VIII. beobachtet. Von der Varietät 2 i von Espirito Santo. 
Anthidium albopilosum n. sp. or or $^{\text {T }}$.

Dem $A$. portoi äufserlich sehr ähnlich, aber + mit unbewehrtem Kopf, $\sigma^{7}$ mit schnabelartigem Segment 7, Beine I und II lang weifs behaart.

․ Schwarz, kurz gelblich befilzt, Kopf und Thorax fein runzlig punktiert, Gesicht schwarz bis auf den gelben Fleck im untern Nebengesicht, Clypeus etwas verlängert, gerundet und am Ende ganz schwach ausgebuchtet, Antenne schwarz, Hinterkopf breit gelb bandiert, Calli h. gelb gefleckt; Scutellum nach hinten dachartig verlängert, gerade abgestutzt, braun gerandet, davor mit breiter, gelber Binde auf der Scheibe; Area glatt, glänzend, mit gerunzeltem Basalstreifen. Abdomen schwarz, schwach braunfilzig, dicht runzlig punktiert, matt, Segment 1-2 ganz schwarz, am Endrande schmal braun, 3-6 gelb mit breiten, braunen Rändern, 4-6 jederseits nach hinten in stumpfen oder spitzen (sechsten) Zahn verlängert, Scopa gelblich. Beine schwarz, Tibien der Beine I und II vorne breit gelb gestreift. Flügel gelblich, mit dunkelbraunem Spitzenrand, Adern gelb, Tegulae braun, gelb gefleckt. - L. $7-7^{1} / 2 \mathrm{~mm}, \mathrm{Br} .3^{1 / 2} \mathrm{~mm}$.

$\sigma^{7}$ wie $q$, aber Gesicht samt Mandibel bis zu den Antennen gelb, Segment 6 lang, abgestutzt, schwach gekielt mit 2 braunen Flecken auf der Scheibe, siebenter schnabelartig nach unten umgebogen, gekielt, gelb und braun gerandet; Ventralsegment braun, jederseits weifs bebüschelt, 6 mit 2 grofsen, gelben Höckern (Kielen) auf der Scheibe. - L. $7-7 \frac{1}{2} \mathrm{~mm}$, Br. $3 \frac{1}{2} \mathrm{~mm}$.

$\sigma^{7} q$ einzeln von Obidos (Amazonas), wo A. D u ck e (Nr. 351) sie am 29. VII. sammelte.

Brasil.

Anthidium pygmaeum n. sp. ㅇ.

Wie $A$. arenarium Ducke, aber Kopf und Thorax viel grober runzlig-punktiert, Segment $3-5$ mit schmalen, gleichbreiten Binden.

9. Schwarz, sparsam weifslich behaart, Kopf und Thorax dicht und sehr grob runzlig-punktiert, matt, Clypeus normal, jederseits gelb gefleckt, Nebengesicht bis fast zum Scheitel gelb; Antenne rotbraun; Hinterkopf gelb gerandet und Mesonotum jederseits vorne, Calli h. gelb gefleckt, Scutellum und Nebenschildchen gelb; Area ganz abweichend vom A. arenarium gebildet: Basalstreifen abgesetzt, horizontal vorstehend und tief längsfurchig, sonst Area fein gerunzelt und matt. Abdomen dicht punktiert, Segment 1-2 jederseits am Rande gelb gefleckt, $3-5$ mit schmaler, gelber Binde, 6 schwarz, Scopa weifs. Beine rostrot, 
III schwarz. Flügel gebräunt, Adern und Tegulae gelbbraun. L. $5 \mathrm{~mm}$, Br. $1^{1 / 4} \mathrm{~mm}$.

o einzeln von Para, wo D ucke (Nr. 335) sie am 24. IX. und 30. VII. beobachtete.

Brasil.

Ceratina virescens n. sp. or 우.

Eine kleine C. laeta Spin., aber Mandibel weifs, im $q$ Clypeus mit feinlinigem, weifsem $\perp$-Fleck, im $\sigma^{7}$ Labrum, Clypeus und Fleck des Nebengesichtes weils.

․ Hellgrün, stark glänzend wie C. laeta, aber Kopf und Thorax fein punktiert, Labrum braun, behöckert, Mandibelbasis weifs, Ende braun; Clypeus mit feinem, weifsem 1-Fleck, Nebengesicht oft mit kleinem, weifsem Fleck; Antenne schwarz. Mesonotum - Mitte glatt und glänzend, Metanotum und Area fein gerunzelt und matt. Abdomen grün bis blaugrün, Endrand und Basis der Segmente schwarz; Ventralsegmente ebenso, lang weifslich behaart. Beine braun, weifs behaart. Flügel bräunlich getrübt, Adern und Tegulae braun. - L. $5 \mathrm{~mm}, \mathrm{Br} .1 \mathrm{~mm}$.

q var. Gröfser, Segmentrand und Basis nur sehr schmal schwarz gefärbt, Antenne braun. - L. $5^{1 / 2}-6 \mathrm{~mm}$, Br. $1^{1 / 4}$ bis $1 \frac{1}{2} \mathrm{~mm}$. var. alpestre $\mathrm{n}$. var. Tarata in Bolivia.

$\sigma^{1}$ ) wie + , aber Labrum, Mandibel und Clypeus ganz elfenbeinweifs, Nebengesicht mit ebensolchem Fleck unten am Rande, Segment 7 einfach gerundet ohne Bewehrung. Kniee und Tibie I vorne weifs, Ventralsegment 7 in kleinen Dorn auslaufend. L. $5 \mathrm{~mm}$, Br. $1 \mathrm{~mm}$.

or + mehrfach von Manatee (Brit. Honduras), wo sie im Februar gefangen wurden.

$$
\text { Ceratina sericea n. sp. } \text {. }
$$

Grölste amerikanische Art, schwarz mit Bronzeglanz, Segment 4-6 goldig glänzend behaart.

․ Schwarz mit Bronzeschimmer, Kopf und Thorax grob runzlig-punktiert, matt, Clypeus schwarz, mit stark aufgebogenem Endrand, jederseits davon auf Nebengesicht ein rundlich gelber

1) Hier mag gleich hervorgehoben werden, dafs die Bildung des siebenten Segmentes bei den $\sigma^{\top}$ von Ceratina-Arten der neotropischen Region in keiner Weise jene hervorragende Mannigfaltigkeit bei den einzelnen Arten zeigt, wir wir sie bei den Formen der alten Welt so schön (vergl. Friese, Bienen Europas, vol. 6, und Friese, Bienen Afrikas, p. 190-201) ausgeprägt finden. Es herrscht bei den amerikanischen Arten durchweg eine einheitliche, und zwar einfache Rundung des Analsegmentes vor, die nur ausnahmsweise noch durch besondere Bildung der Ventralsegmente gestützt wird. 
Fleck, Labrum schwarz, stark höckerig, mitten an der Basis mit mächtigem, glattem Kiel; Mandibel zweizähnig, mit breiter, höckeriger Basis, äufsere Orbita mit langem, gelbem Fleck; Antenne schwarz, zweites Geifselglied $=3+4$, Pronotum gelblich, seidenglänzend behaart; Mesonotum vorne mit 3 eingedrückten Längslinien und glatter Scheibe, Scutellum grob punktiert, Metanotum und Area fein gerunzelt, matt. Abdomen fein und runzligpunktiert, Segment 1 kahl mit glatter Basis, 2-3 schwach seidenartig behaart, 4-6 dicht goldglänzend seidenartig behaart, 6 mit Längsfurche, mitten zugespitzt. Ventralsegmente punktiert, 1-3 mit schwarzem, halbrundem, fein runzlig-punktiertem Fleck, 4-6 goldglänzend, grob punktiert, 6 in Dorn endend. Beine schwarz, Tarsen braun, weifslich behaart, Scopa weifslich, Calcar rotbraun. Flügel gelblich getrübt, mit dunklem Endrande, Adern rotgelb, Tegulae schwarzbraun, glatt, Vorderhälfte braun behaart. L. $13 \mathrm{~mm}$, Br. $4 \mathrm{~mm}$.

$1 q$ von Villa Rica (Paraguay), Burgdorff leg.

\section{Ceratina dimidiata n. sp. ㅇ.}

Mittelgrofse Art, Kopf und Thorax schwarz, hintere Thoraxwand blau, Abdomen goldig bronziert.

9. Schwarz, kaum greis behaart, Kopf rundlich und wie der Thorax grob punktiert, glänzend; Clypeus mit $\perp$-förmigem, gelbem Fleck, Labrum grob runzlig-punktiert, mit glatten Höckern und gelblichem Haarschopf am Ende; Mandibel glatt, zweizähnig, mit stark verbreitertem Basalteil, Antenne schwarz, zweites Geifselglied wenig länger als 3. Mesonotum einzeln punktiert, mit $\tilde{5}$ eingedrückten Längslinien, glänzend, Scutellum und Metanotum feiner und dichter punktiert, mit Bronzeglanz, Area schön blau, längsrunzlig und die Runzeln wieder fein quergerunzelt, matt. Abdomen punktiert, glänzend, bronzefarbig, 4-6 goldgelb, grob gerunzelt, 6 in kleine Spitze verlängert. Ventralsegmente grob punktiert, lang goldig behaart, 1-3 mit kleiner, schwarzer, mattpunktierter Basalplatte. Beine schwarz, weifslich behaart, Scopa weifs, Calcar schwarz mit rotem Ende. Flügel getrübt, Adern und Tegulae schwarzbraun, Vorderhälfte der Tegulae behaart. L. $10 \mathrm{~mm}$, Br. $3 \mathrm{~mm}$.

2 q von San José in Costa Rica, Burgdorff leg. America centr.

$$
\text { Ceratina laticeps n. sp. ㅇ. }
$$

Durch die dunkle Erzfarbe und den breiten Kopf auffallend. ๆ. Schwarz, mit dunkel erzfarbenem Glanz, kaum noch gelblich behaart, Kopf breiter wie lang, viel breiter als der Thorax, 
mit einzelnen groben Punkten, Zwischenräume der Punkte so grols wie die Punkte, glatt und glänzend; Clypeus schwarz, mit grofsem, gelbem Scheibenfleck, der unten etwas verbreitert ist, Labrum schwarz, runzlig-punktiert, vor dem Endrand mit 2 grofsen Höckern, Mandibel schwarz, mit rotem Ende; Antenne schwarz, Scapus und Geifselglied 1 rot. Mesonotum mit einzelnen feinen und groben Punkten, sonst spiegelglatt, die 5 eingedrückten Längslinien kaum erkennbar, Scutellum einzeln grob punktiert, Pleuren grob und dicht punktiert, Metanotum fein gerunzelt, matt, Area fein längsrunzlig, ebenfalls matt. Abdomen dicht punktiert, Segment 1 glatt, 5-6 dicht runzlig-punktiert, matt, 6 in grolse Spitze verlängert, Ventralsegmente punktiert. Beine schwarz, Tarsen braun, rotgelb behaart, Scopa gelblich, unten weifs, Calcar braun. Flügel gelblich getrübt, der Vorderrand breit, wie die Radialzelle dunkel braun, Adern und Tegulae braun. L. $9 \mathrm{~mm}$, Br. $21 / 2 \mathrm{~mm}$.

$q$ von San José (Costa Rica), Burgdorff leg.

\section{Ceratina wagneri n. sp. ㅇ.}

Der C. laticeps n. nahestehend, aber Flügel gelblich. Abdomen grün.

․ Schwarz mit Bronzeglanz, Kopf und Thorax sparsam punktiert, Clypeus mit rundlich gelbem Scheibenfleck, sonst fast glatt, mit deutlich erhabenen Rändern, Labrum uneben, undeutlich punktiert, mit 2 Basalbeulen, Mandibel schwarzbraun, mit breiter, glatter Basis. Antenne schwarz, unten braun, zweites Geifselglied $=3+4$. Mesonotum fast glatt, glänzend, hintere Thoraxwand grünlich, lang und einzeln gelbbraun behaart, Area längsrunzlig, fast matt. Abdomen erzgrün, fein punktiert, 5-6 dicht höckrig gerunzelt, 6 in eine lange, dreieckige Spitze ausgezogen. Ventralsegmente grob punktiert, 2-3 mit schwarz halbkreisförmigem, quergerunzeltem Basalfleck. Beine schwarz, Tarsen braun, lang gelbbraun behaart, Scopa gelblich, Kniee mit gelbem Fleck, Calcar rotgelb. Flügel stark gelblich getrübt, Adern gelbbraun, Tegulae schwarzbraun. - L. $10 \mathrm{~mm}, \mathrm{Br} .2^{1} / 2 \mathrm{~mm}$.

1 \& von der Sierra Durango in Mexico.

Herrn Prof. Dr. Franz v. Wagner in Graz zu Ehren benannt.

Ceratina lehmanni n. sp. ㅇ.

Eine sehr buntgefärbte Art, die durch die purpurrote Färbung von Kopf und Thorax auffällt.

\%. Kopf rot bronziert, Thorax purpurrot glänzend, Abdomen erzgrün; Kopf grob runzlig punktiert, Clypeus mit breitem, gelbem 
Querfleck, Nebengesicht breit gelb bis zur Antennenbasis, auch äufsere Orbita breit gelb, Labrum schwarz, stark konvex, fast halbkuglig mit 2 Basalbeulen und 2 Längsrippen, sonst runzlig punktiert, am Ende mit gelbem Haarpinsel; Antenne schwarzbraun, erstes Geifselglied rot. Thorax grob punktiert, Mesonotum mit schwarzer, glatter Scheibe, Metanotum und Area feiner gerunzelt, tief purpurrot. Abdomen grob punktiert, Ventralsegmente feiner. Beine schwarz, Tarsen braun, lang weifs behaart, Knie I und Tibien I vorne gelb gefärbt, Calcar rot. Flügel stark gebräunt, Adern und Tegulae braun. - L. $7^{1 / 2} \mathrm{~mm}$, Br. $21 / 4 \mathrm{~mm}$. 1 q von Popayan (Columbia), Lehmann leg.

\section{Ceratina versicolor n. sp. q.}

In Form und Farbe der C. lehmanni ähnlich, aber Thorax blau, Körper viel grober skulpturiert, Clypeus mitten tief gefurcht, ohne gelbe Zeichnung.

․ Kopf rötlich bronziert, Thorax blau mit schwachem Erzschimmer, Mesonotum schwarz und erzfarben, Abdomen erzgrün, wie lehmanni, aber Clypeus ohne Gelb, mit tiefer, glatter Mittelfurche und erhabenen Furchenrändern, Labrum schwarz, schwach konvex, Nebengesicht mit gelbem Endfleck; Antenne schwarz, Geifselglied 1-2 braun. Mesonotum noch grober als bei lehmanni punktiert, mit 2 tiefen eingedrückten Längslinien, Scutellum sehr grob runzlig-punktiert, Pleuren und hintere Thoraxwand blau, Metanotum und Area fein gerunzelt, matt. Abdomen erzgrün, Segment 1 glatt, schwarzbraun, 2-3 mit schwarzbrauner Endhälfte, sonst sparsam punktiert, 4-6 runzlig-punktiert; Ventralsegmente erzgrün, mit braunen Segmenträndern. Beine schwarz, Tarsen braun, weifslich behaart, Scopa gelblich, Calcar schwarz, mit hakenförmig gebogenem Ende. Flügel stark gebräunt, Radialzelle schwarzbraun, Adern und Tegulae schwarzbraun. -- L. $9 \mathrm{~mm}$, Br. $3 \mathrm{~mm}$.

q am 14. VIII., 1902 bei Para durch A. D u cke gefangen. Brasil.

$$
\text { Ceratina duckei n. sp. or }
$$

Wie C. bicolorata Sm., aber hell erzgrün, + im Gesicht mit nur 3 gelben Flecken, Clypeus einfach gewölbt, ohne eingedrückte Mittelfurche; Flügel braun.

․ Erzgrün, kaum behaart, Kopf und Thorax grob punktiert, nur oben im Gesicht gerunzelt; Clypeus gewölbt, ohne Eindruck, punktiert und äufserst fein gerunzelt, Endrand mit kleinem, gelbem Fleck, Nebengesicht unten in der Ecke gelb, an äufserer Orbita mit gelbem Streif, Mandibel, Labrum und Antenne schwarz, letztere 
unten braun. Mesonotum mit grofser, glatter, schwarzer Scheibe, Scutellum, Area und Abdomen wie bei C. brunneipes n. (s. u.) skulpturiert. Beine schwarz, weifslich behaart, Scopa weifslich, Calcar rot, Kniee I gelb. Flügel braun, Adern und Tegulae schwarzbraun. - L. $7^{1 / 2}-8^{1 / 2} \mathrm{~mm}, \mathrm{Br} .2^{1 / 2} \mathrm{~mm}$.

$\sigma^{\top}(?)$ wie $q$, aber Clypeus mit feiner, weifsgelber Mittellinie, Labrum mit winzig kleinem Fleck; Segment 7 gerundet, kaum noch ausgebuchtet. - L. $8 \mathrm{~mm}$, Br. $2^{1} / 2 \mathrm{~mm}$.

$2 \sigma^{7}, 12$ qon Para (Mai - Juni), Macapa, Marajo, Mazagao und von Obidos (Januar) von $\mathrm{D} \mathrm{u} \mathrm{cke}$ erhalten; $q=$ Nr. 272 und $273, \sigma^{\top}=$ Nr. 275 .

$\sigma^{7}$ fraglich, ob hierher gehörend, $\sigma^{7} q$ wurden nur zusammen gefangen!

$$
\text { Ceratina portoi n. sp. } \text { ․ }
$$

Wie C. duckei, aber dunkel erzfarben, Gesicht mit 3 gelben Längslinien, Clypeus mit breiter, gelber Mittellinie, und die beiden inneren Orbitae bis über die Antennen gelb; Mesonotum schwarz mit glatter Scheibe. Abdomen dunkler, feiner skulpturiert. Beine schwarz, Scopa weifs. - L. $8^{1} / 2 \mathrm{~mm}$, Br. $2 \mathrm{~mm}$. Auffallend ist auch die lange Zunge (Glossa), die $7 \mathrm{~mm}$ Länge erreicht.

q von Para, am 22. III. 1900 gefangen und von $\mathrm{D} \mathrm{u} c \mathrm{ke}$ mir mitgeteilt.

Herrn Dr. Porto in Para zu Ehren benannt.

Ceratina darwini n. sp. or

C. darwini fällt im $\sigma^{\top} q$ durch die aufserordentlich grob runzlige Skulptur des Abdomen auf, vor dem Endrand der Segmente 2-5 tritt eine $\mathrm{g} \mathrm{r}$ o be Punktreihe deutlich hervor.

․ Erzgrün, sehr grob skulpturiert, Kopf runzlig-punktiert, Clypeus mit glattem Mittelfeld und schwacher Längsfurche, Endrand schwarz, glatt, und mitten mit kleinem, gelbem Fleck, Labrum und Mandibel schwarz, Nebengesicht mit gelbem Fleck in der unteren Ecke, äufsere Orbita mit gelbem Längsstreifen. Thorax grob punktiert, Punktränder erhaben; Mesonotumscheibe fast glatt; Metanotum und Area fein gerunzelt, fast matt. Abdomen punktiert, Scheibe von Segment 2-3 sparsam und fein punktiert, $4-6$ sehr grob und höckerig gerunzelt, $1-5$ vor dem Endrand mit einer groben, unregelmälsigen, aber deutlich erkennbaren Punktreihe, die auf $2-3$ am deutlichsten hervortritt; Ventralsegmente flach punktiert, mit schwarzen Endrändern, 6 gekielt. Beine schwarz, Tarsen braun, weifslich behaart, Scopa gelblich, Knie I und Tibie I gelbgefleckt, Calcar rot. Flügel 
getrübt, mit dunklerem Spitzenende, Adern braun, Tegulae schwarzbraun. - L. $7-8 \mathrm{~mm}$, Br. $2 \mathrm{~mm}$.

$\sigma^{7}$ wie $q$, besonders auch die Punktreihe auf Segment 2-4 recht deutlich, aufser den 3 gelben Gesichtsflecken des $q$ finden sich noch Labrum und Mandibelbasis gelb gefleckt, Segment 7 nach unten kapuzenartig erweitert, stark gekielt, fast glatt, von oben gesehen = einfach verjüngt verlaufendes Analsegment, Ventralsegmente fein punktiert. Beine braun. L. $6^{1} / 2 \mathrm{~mm}$, Br. $2 \mathrm{~mm}$.

$2 \sigma^{\top}$ und 20 o 9 vara, Macapa und Obidos meist im November und Januar von $\mathrm{D} \mathrm{u} \mathrm{ck}$ e gesammelt, aber auch einzeln im April und September.

$$
\left(q=\text { Nr. } 287 \text { und } 290 ; \sigma^{T}=291\right. \text { und 292.) }
$$

\section{Ceratina huberin. sp. or + .}

Durch das dunkle Gesicht unter allen Arten Brasiliens auffallend, beim $q$ nur je ein kleiner Fleck auf dem Nebengesicht, beim $\sigma^{7}$ mit ganz schwarzem Gesicht, Segment 7 gerundet.

․ Erzgrün, wie C. darwini, aber etwas feiner skulpturiert, Clypeus mit deutlich glatter Mittelfurche, sonst punktiert und ohne Spur von gelber Zeichnung, Nebengesicht mit winzig kleinem, gelbem Fleck am unteren Ende; Mesonotum mit glatter, erzgrüner Scheibe; Area fein längsrunzlig. Abdomen auf Segment $1-3$ punktiert, $4-6$ mehr körnig gerunzelt. Beine schwarz, Knie I und Tibie I mit gelbem Fleck, Scopa gelblich. Flügel gelblich getrübt, mit dunklem Fleck am Spitzenrand, Adern braun, Tegulae schwarzbraun. - L. $6{ }^{1} / 2-7 \mathrm{~mm}, \mathrm{Br} .1^{3} / 4 \mathrm{~mm}$.

$\sigma^{7}$ wie $q$, aber Gesicht ganz einfarbig erzgrün, innere Orbitae nach vorne konvergierend, Clypeus mit kräftiger Mittelfurche. Thorax sehr grob punktiert. Abdomen meist gerunzelt, Segment 7 gerundet, auf der Ventralseite liegend; Ventralsegment 6 mit rotfilzigem Endrand. - L. $6^{1} / 2 \mathrm{~mm}$, Br. $1^{1} / 2 \mathrm{~mm}$.

2 o $3 \sigma^{7}$ von Para, am 2. III. und 25. XI. von D ucke gefangen, Nr. 293 (\$), 294, 295 und $296\left(\sigma^{7}\right)$.

Herrn Dr. Huber, Direktor des "Museu Göldi“ in Para gewidmet.

$$
\text { Ceratina brunneipes n. sp. ơ ㅇ․ }
$$

Wie C. bicolorata Sm., aber hell erzgrün, Gesicht reichlich gelb gezeichnet, Clypeus ohne Mittelfurche, Beine reichlich braun gezeichnet.

․ Erzgrün, sparsam weils behaart, Kopf und Thorax grob runzlig-punktiert, Labrum, punktiert, wie Mandibel schwarz, Clypeus mit groben Punkten, aber diese Skulptur äufserst fein 
querrunzlig, ohne Mittelfurche, Endrand schwarz mit gelbem Fleck, Nebengesicht breit gelb bis zu den Antennen hinauf, äufsere Orbita mit breitem, gelbem Streifen; Antenne schwarzbraun; Mesonotum mit schwarzer, glatter Scheibe, Scutellum punktiert, Metanotum und Area fein gerunzelt, matt. Abdomen grob punktiert, auf der Scheibe der Segmente sparsam, Segment 5-6 fast grob gerunzelt, 6 mit eingedrückter Mittellinie, Ende in kleinen Dorn verlängert. Ventralsegmente punktiert, breit hellbraun gerandet, 2-3 mit halbkreisförmigem, schwarzem, mattem Basalfleck. Beine braun, Kniee von Beine I und II gelb gefleckt, Tibie I gelb gestreift, weifslich behaart, Scopa weifs. Flügel gelblich getrübt, mit dunklem Endrand, Adern braun, Tegulae rotbraun. L. $8-10 \mathrm{~mm}$, Br. $2^{1} / 2-3 \mathrm{~mm}$.

$\sigma^{7}($ ?) wie + , aber Mandibelbasis, Labrum und Endrand vom Clypeus breit gelb, Segment 7 gerundet, schwach ausgebuchtet; Ventralsegment 6 mit kleiner Grube vor dem Ende, die lang rotgelb behaart ist. - L. $8 \mathrm{~mm}$, Br. $2^{1 / 2} \mathrm{~mm}$.

3 ơ $^{7}, 10 q$ von Obidos, Para, Macapa (Amazonas) und von S. Cruz (in S. Brasil) im Januar, April, Mai und Juni von D u cke gesammelt.

$\left(\sigma^{\top}=\right.$ Nr. $270, q=$ Nr. 268.)

Es ist nicht erwiesen, dafs das $\sigma^{7}$ sicher hierzu gehört; sie flogen nur zusammen.

Ceratina punctiventris n. sp. ㅇ.

C. punctiventris fällt durch ihre Grölse und die schwarzblaue Färbung auf, Abdomen sparsam und grob punktiert, besonders auf Segment 2-4, Gesicht mit 5 gelben Flecken.

․ Schwarzblau, stellenweise mit blaugrünem Schimmer, gröfste Art vom Para-Gebiet. Kopf und Thorax grob runzligpunktiert, Clypeus so lang wie breit, äufserst fein runzlig-punktiert, mit einzelnen gröfseren Punkten, am Vorderrand mit gelbem Fleck und schwacher Mittelfurche; Nebengesicht mit je 2 gelben Flecken, der eine am Ende, der andere in Antennenhöhe, Labrum und Mandibel schwarz, Antenne schwarzbraun, hinter der äufseren Orbita ein gelber, ziemlich breiter Streifen. Mesonotum mit 2 glatten, schwarzen Flächen auf der Scheibe, Area fein quergerunzelt, an der Basis fein längsrunzlig. Abdomen punktiert, auf Segment 1-3 auf der Scheibe sehr sparsam, 4-6 grob gerunzelt. Ventralsegmente punktiert, mit schwarzen Endrändern. Beine schwarz, Tarsen braun, weifs behaart, Scopa rein weifs; Kniee I weifs gefleckt, Tibie I weifs gestreift, Calcar rotbraun. Flügel getrübt, mit dunklem Endrand, Adern und Tegulae braun. L. $9-10 \mathrm{~mm}$, Br. $3 \mathrm{~mm}$. 
$\sigma^{7}$ unbekannt, trotz der zahlreichen + . $q$ zahlreich von Para durch $\mathrm{D} \mathrm{u} \mathrm{cke}$ erhalten; fast in allen Monaten des Jahres von ihm beobachtet.

Brasil.

$$
\text { Ceratina muelleri n. sp. } \quad \text { ․ }
$$

Eine der kleinsten Arten von der Gröfse der C. oscalidis Schrottk. und C. minima Friese, aber Antenne und Beine braun, innere Orbita mitten meist mit weifsem Fleck.

․ Dunkelblau, stark glänzend, Kopf, viereckig, glatt, Clypeus mit weifsem, eckigem Längsfleck, Labrum, Mandibel und Antenne braun, Fühlergruben sehr tief liegend, jederseits davon am inneren Augenrande ein weifser Fleck, ein länglicher Fleck längs der äufseren Orbita; Gesicht beulig erscheinend. Thorax einzeln punktiert, Mesonotum mit 3 eingedrückten Längslinien, mit glatter Scheibe, Scutellum meist glatt, Metanotum und Area dicht und fein gerunzelt, matt. Abdomen einzeln und undeutlich punktiert, Segment 1-3 braun durchscheinend, 4-6 blau; Ventralsegmente meist braun. Beine braun, weifslich behaart. Flügel hyalin, mit dunklem Rande, Adern braun, Tegulae gelbbraun. - L. $3^{1 / 2}-4 \mathrm{~mm}$, Br. ${ }^{3} / 4 \mathrm{~mm}$.

$q$ mehrfach im Februar bis April bei Para und im Juli bei Obidos, D u ck e leg.

Amazonas (Brasil).

q var. Kopf, aufser Clypeus, blau ohne weifse Flecken.

var. immaculata n. var.

Itaituba im VIII. und Para im II. und IV.

Ceratina combinata n. sp. or.

$\sigma^{7}$ wie C. muelleri und wohl das or dazu, Clypeus und Labrum fast ganz weifs und auch ein Fleck daneben auf dem Nebengesicht; Abdomen fast schwarz, Segment 6 gerundet, unbewehrt. L. $3 \mathrm{~mm}, \mathrm{Br} .{ }^{3} / 4 \mathrm{~mm}$.

$1 \sigma^{7}$ von Para am 17. IX. von $\mathrm{D} \mathrm{u} \mathrm{cke}$ gesammelt.

$$
\text { Ceratina herbstin. sp. or 오. }
$$

Wie C. postica Spin., aber viel kleiner, mit ganz schwarzem Abdomen, $\sigma^{7}$ mit ganz gelbem Gesicht.

ㅇ. Schwarz, spärlich weifs behaart, Kopf und Thorax fein und sparsam punktiert, mit glatten, glänzenden Zwischenräumen; Gesicht flach, Clypeus gerade abgestutzt; Area gewölbt, glatt. Abdomen undeutlich punktiert, glänzend. Ventralsegmente ebenso, braun gerandet. Beine schwarz, weifs behaart, Scopa lang und 
weifs. Flügel hyalin, Rand kaum dunkler, Adern schwarzbraun, Tegulae schwarz. - L. $5-5 \frac{1}{2} \mathrm{~mm}, \mathrm{Br} .11 / 2 \mathrm{~mm}$.

$\sigma^{7}$ wie $q$, Gesicht bis zu den Antennen gelb, auch Labrum gelb. - L. $5 \mathrm{~mm}$, Br. $1 \frac{1}{2} \mathrm{~mm}$.

$\sigma^{7}+9$ zahlreich im September und im Februar bei Concepcion durch P. Herbst gefangen; eine biologische Notiz verdanke ich dem Entdecker, „am 21. VIII. 04 in ausgehöhltem, trockenem ,avellano'-Zweig". Sonst fliegt C. herbsti auf Disteln.

$\mathrm{Ob}$ die Art mit Halictus gayatinus Spin. $q$ identifiziert werden kann, ist wohl sehr fraglich?

\section{Ceratina humboldti n. sp. or $\sigma^{T}$.}

Der C. aspera Schrottk. nahestehend, aber Segment 7 gerundet, schmal ausgebuchtet.

․ Blau, sparsam weifslich behaart, Kopf runzlig-punktiert, Clypeus fast mit Längsrunzeln, Endrand mit kleinem, gelbem Querfleck, Nebengesicht auch unten mit kleinem, gelbem Längsfleck, ebenso die innere Orbita (also 5 kleine Gesichtsflecken), Labrum stark gewölbt, mit einzelnen grofsen Punkten, nebst den Mandibeln schwarz, Antenne schwarzbraun, äufsere Orbita mit kleinem, gelbem Längsstrich. Thorax grob punktiert; Mesonotum auf der Scheibe mit glatten Stellen und 3 deutlich eingedrückten Längslinien; Area fein längsrunzlig. Abdomen etwas dunkler blau, punktiert, Segment 5-6 gerunzelt. Ventralsegmente punktiert, Analsegment gekielt. Beine schwarz, Tarsen braun, Scopa weifs, Calcar rot. Flügel getrübt, mit braunem Endrand, Adern braun, Tegulae schwarzbraun. - L. $7-8 \mathrm{~mm}$, Br. $2 \mathrm{~mm}$.

$\sigma^{7}$ wie $q$, Antenne etwas länger, an der inneren Orbita kann der gelbe Fleck auch fehlen, Segment 7 gerundet und schwach ausgebuchtet; Ventralsegment 6 mitten am Rande eingedrückt, braunfilzig behaart. - L. $6{ }^{1} / 2-8 \mathrm{~mm}$, Br. $1 \frac{1}{2}-2 \mathrm{~mm}$.

$2 \sigma^{7}, 2 q$ von Jundiahy im Oktober, November und im Januar von $\mathrm{Schrottky}$ gesammelt.

Sao Paulo (Brasil).

Ancyloscelis Latr.

1825. Ancyloscelis Latreille. - Fam. nat. règn. anim. p. 363.

1853. Ptilothrix Smith. - Cat. Hym. Brit. Mus. I. p. 131.

1879. Diadasia Patton. - Bull. U. St. Geol. Surv. V. p. 475.

1879. Emphor Patton. - Bull. U. St. Geol. Surv. V. p. 476.

1903. Thygater Holmberg. - Ann. Mus. Buenos Aires IX. p. 385.

1903. Teleutemnestra Holmberg. - Ann. Mus. Buenos Aires IX. p. 400. 1908. Dipedia Friese. - Apidae v. Argentina. p. 52.

Die Klarstellung der beiden Gattungen Thygater und Teleutemnestra verdanke ich Herrn K. Schrottky in Puerto Bertoni, 
von dem wir auch wohl bald die Kenntnis der meisten Arten aus Holmbergs Arbeit erwarten dürfen. Ob wir die einzelnen Genera bestehen lassen oder zusammenwerfen, dürfte gegen die Hervorhebung der natürlichen Verwandtschaft nicht von Belang sein. Jedenfalls scheint mir die Betonung der Verwandtschaft vorläufig das Wichtigste in der ganzen Systematik zu sein, sonst werden sich die Autoren nur schwer aus ihren Arbeiten verstehen können.

Ancyloscelis plumata $\mathrm{Sm}$.

1853. Ptilothrix plumata Smith, q. - Cat. Hym. Brit. Mus. I. p. 132; t. 6. f. $11-14$.

1879. Diadasia Patton. - Bull. U. St. Geol. Surv. V. p. 475.

1879. Emphor Patton. - Bull. U. St. Geol. Surv. V. p. 476.

1903. Teleutemnestra relata. - Ann. Mus. Buenos Aires IX. p. 401.

$\sigma^{7}$ wie + , auch Kopf und Thorax gelbbraun behaart, die Beine rot wie beim $q$, aber sehr verlängert, besonders die Beine III. L. $10-10^{1} / 2 \mathrm{~mm}$, Br. $4 \mathrm{~mm}$.

Besonders in Nord- und Ost-Brasilien heimisch. Ich erhielt gut mit der Beschreibung von S mith übereinstimmende Exemplare von Para, wo Ducke sie im Mai fing; auch von Jundiahy, wo Schrottky sie am 12. II. beobachtete.

Die Exemplare von Paraguay, Argentinien, Ecuador, also aus dem Westen, haben schwarze Beine, ich trenne sie als var. nigrescens $\mathrm{n}$. var. ab.

Die Exemplare vom Süden, d. h. von Salta und Cordova (Argentina), haben wohl schwarze Beine, aber rote Antennen und aufserordentlich breite, gelbe Segmentbinden, die mitunter das ganze Abdomen gelb tomentiert erscheinen lassen. Die gelben Binden erreichen den Seitenrand. Gröfste Form. - L. 11-12 mm, Br. $4^{1} / 2-5 \mathrm{~mm}$. var. ruficornis $\mathrm{n}$. var. $\sigma^{7}+$ von Cordova und Salta.

Ancyloscelis (Ptilothrix) nigrita n. sp. or ․

Der A. plumata Sm. (= ? Teleutemnestra relata Holmbg.) sehr ähnlich und event. als melanotische Form davon aufzufassen, aber durchweg schwarz statt braungelb behaart.

․ Schwarz, schwarz behaart, Kopf und Thorax punktiert, Clypeus doppelt so breit wie lang, grober punktiert, Zwischenräume der Punkte gröfser als diese, Labrum in Spitze ausgezogen, mit gewulstetem Endrand, Mandibel schwarz, mit roter Mitte und gelbem Ende; Antenne schwarz, keulenförmig, unten braun, zweites Geifselglied fast länger als $3+4$. Mesonotum sparsam punktiert, glänzend, Zwischenräume viel grölser als die Punkte; Area fein punktiert, kaum gegen die Umgebung abstehend. Abdomen fein, netzartig punktiert, glänzend, fein schwarz behaart, 
Segment 1 jederseits am Rande weifs bebüschelt, $2-4$ am Endrande mit breiter, gelbfilziger Binde, die aber den Seitenrand bei weitem nicht erreicht, die gelben Haare sind fast schuppenartig (viermal so lang wie breit), 5-6 schwarz behaart, 6 mit dreieckiger, quergerunzelter Analplatte; Ventralsegmente 2-6 lang weifs bebürstet, wie eine Scopa bei den Bauchsammlern. Beine schwarz, schwarzbraun, Scopa dünn, schwarz, Tibienende weifs bebüschelt, Metatarsus lang schwarz beborstet. Flügel gelblich, Adern gelbbraun, Tegulae schwarzbraun. - L. $9 \mathrm{~mm}$, Br. $3 \frac{1}{2} \mathrm{~mm}$.

var. ㅇ. - Binden auf Segment $2-4$ in je 2 rundliche Flecke aufgelöst; Thoraxhaare mit graugelben vermischt, Tegulae gelbbraun. - L. $9^{1} i_{2} \mathrm{~mm}$, Br. $4 \mathrm{~mm}$. var. sexmaculata $\mathrm{n}$. var. 1 o von Bolivia.

$\sigma^{7}$ wie $q$, aber Segment $2-5$ mit gelblicher Binde, 6-7 schwarz, 7 zweispitzig, Ventralsegmente $2-4$ weifs beborstet, 5 mitten glatt, braun, jederseits schwarz bebüschelt, 6 dachförmig gekielt, schwarzborstig, Tegulae gelbbraun. - L. $9 \mathrm{~mm}$, Br. $3 \frac{1}{2} \mathrm{~mm}$.

$\sigma^{7} q$ von Tarata (Bolivia), $q$ auch von Salta (Argentina).

Ancyloscelis bombiformis Patt.

1878. Melissodes bombiformis Cresson, oㅇ․ - Porc. Acad. Nat. Sc. Philadelphia 1878. p. 219.

1879. Emphor bombiformis Patton, ơ ㅇ. - Bull. U. St. Geol. Surv. V. p. 476.

1896. Eucera bombiformis Dalla Torre. - Cat. Hym. X. p. 227.

"ㅇ. - Black; head and thorax clothed with short ochraceous or fulvo-ochraceous pubescence, sparse on face; vertex shining; clypeus sparsely punctured, nude on the disk; pubescence of thorax very dense; tegulae fulvo-testaceous; wings fuscous, paler on apical margin, nervures black, 2. submarginal cell about $2 / 3$ the length of 1 ., narrowed above, the 3 . shorter than 1. , rounded at tip and narrowed nearly $1 / 2$ towards marginal; legs piceous, the pubescence black, mixed with pale on anterior pair, that on posterior legs long; abdomen opaque, with very short black or fuscous pubescence, that on 1 . segment sometimes mixed with ochraceous. - Length 55-70 inch (= $=14-18 \mathrm{~mm} \mathrm{lg.).}$

$0^{7}$. - Closely resembles the $q$; clypeus and labrum with short dense ochraceous pubescence; antennae short as in $q$; legs robust, especially the posterior pair, the pubescence very short, black, ochraceous on coxae, trochanters and anterior femora beneath, 4 posterior tarsi long, the basal joint of posterior pair long and curved; basal segment of abdomen clothed with short 
dense ochraceous pubescence, that on venter with black or fuscous. Length 60 inch (=15 mm).

Virginia, Georgia, Kansas.

This has much the appearance of certain species of Bombus." Ancyloscelis bombiformis liegt mir in $1 q$ von Illinois aus der coll. Robertson vor; 2 weitere $q$ von Amerika, die durch das tiefschwarz behaarte Abdomen und hellere Flügel mit dunklerem Rande etwas abweichen.

$$
\text { Ancyloscelis fuliginosa n. sp. or. }
$$

$\sigma^{7}$. Nigra, dense flavido-hirta, abdomine fuliginoso-hirto, capite punctato, clypeo brevi truncataque, grosse punctato, fronte glabro; pedibus prolongatis, tarsorum articulo 1 longissimis inflexisque; alis fuscis.

Ancyloscelis fuliginosa ist an ihrer Gröfse und dem dicht braun behaarten Abdomen kenntlich.

$\sigma^{7}$. Schwarz, Kopf und Thorax dicht gelblich behaart, Abdomen ebenso, aber braun, Segment 1 ist etwas heller behaart mit dunklerer Scheibe; Kopf punktiert, Clypeus grober, die Ocellengegend glatt und glänzend; Fühler schwarzbraun, zweites Geifselglied so lang als das dritte und vierte. Thorax deutlich und ziemlich dicht punktiert, die Skulptur aber infolge der dichten Behaarung schwer erkennbar. Abdomen feiner und dichter punktiert, fast matt, die Segmentränder von 1-4 infolge heller Behaarung auf der Mitte schmal bandiert erscheinend. Ventralsegmente mit blafsgelben Rändern, dicht braun befranst, Segment 6 kielartig erhaben, bürstenartig behaart. Beine schwarzbraun, Aufsenseite fast braun, schwarzbraun behaart, alle verlängert, besonders die hinteren Beine an den Tarsen, das erste Tarsenglied hier gebogen und kaum kürzer als die Tibien, Hinterschenkel und Tibien stark verdickt, Sporen lang; Flügel gebräunt, Adern schwarzbraun, Tegulae gelbbraun mit dunklerer Scheibe, fein und undeutlich punktiert. - L. $18 \mathrm{~mm}$, bei $7 \mathrm{~mm}$ Abdomenbreite.

Von Ancyloscelis fuliginosa verdanke ich $1 \sigma^{7}$ von Attac (Mexiko?) Herrn Dr. A. v. Schulthess in Zürich.

$$
\text { Ancyloscelis hirsuta n. sp. or 우. }
$$

Der A. australis Cr. (Diadasia) nach Grölse und Farbe nahestehend, aber überall viel länger, dichter und struppiger behaart, $\sigma^{7}$ mit am Ende verbreitertem und lappig verlängertem Metatarsus.

․ Schwarz, glatt, glänzend, überall, auch an den Beinen, lang und struppig grauweifs behaart, Kopf und Thorax einzeln und zerstreut punktiert, Antenne sehr kurz, zweites Geifselglied $=3+4$, Endglied plattgedrückt. Area des Thorax fein ge- 
runzelt, mit breiter Mittelfurche. Abdomen undeutlich punktiert, sehr lang und dicht abstehend behaart und dadurch von allen Arten zu erkennen, Segment 5 am Rande und 6 rötlich beborstet, 6 mit matter, gerunzelter Analplatte; Ventralsegmente deutlich punktiert mit bleichen, lang befransten Endrändern, Fransen auf der Scheibe rötlichbraun. Beine schwarz, lang abstehend weifslich behaart, Tibie aufsen mit kurzer, braunfilziger Behaarung, Scopa weifslich, innen gelblich, Metatarsus innen schwarzbraun behaart, Tarsenglied 1 der Beine I und II gebogen, innen dunkel behaart; Calcar gelblich. Flügel hyalin, Adern braun, Tegulae schwarz. - L. $9-10 \mathrm{~mm}$, Br. $3^{1 / 2}-4 \mathrm{~mm}$.

$\sigma^{7}$ wie + , Antenne wenig länger, Segment 5-7 gleichmäfsig lang weifslich behaart, 7 in 2 lange, dünne Dorne endend, die aber durch die lange Behaarung verdeckt werden; Ventralsegmente weifslich behaart, 5-6 rötlich und kürzer gefranst, 6 etwas konkav erscheinend. Beine schwarz, Tarsen verlängert, Metatarsus gekrümmt, am Ende verbreitert und lappig verlängert. L. $8-12^{1 / 2} \mathrm{~mm}$, Br. $3^{1 / 2}-4^{1 / 2} \mathrm{~mm}$; also mit Grölsenunterschieden bei $q$ wie $\sigma^{\top}$ in beträchtlichem Mafse.

$\sigma^{7}+9$ mehrfach von Tarata in Bolivia und von Cuzco in Peru in Höhen von $3200-4200 \mathrm{~m}$ im April bis Juli. Die eigenartig struppige Behaarung weist offenbar, wie bei den hier lebenden Anthophora-Arten, auf kühlere Temperaturverhältnisse hin.

$$
\text { Ancyloscelis duckei n. sp. or ㅇ․ }
$$

Der A. riparia Ducke nahe verwandt, aber grölser, beim $q$ Segment $2-4$ weifshaarig bandiert, Basis von $3-5$ schwarz befilzt, beim $\sigma^{\top}$ mit gekerbten Antennen.

9. Schwarz, weifslich behaart, nur auf Mesonotum und Scutellum gelbbraun behaart, Kopf und Thorax fein punktiert, glänzend, Labrum fünfeckig, mit eigenartig dick gewulstetem Endrand; Area etwas feiner punktiert, aber nur wenig gegen die Umgebung abstechend. Abdomen fein punktiert, glänzend, Segment $1-2$ an der Basis schwach weifsfilzig, 3-4 mit schwarz befilzter Basis, 1-4 mit weifshaariger Randbinde, 5 weifsfilzig, der Endrand vom fünften und das ganze sechste schwarzbraun beborstet; Ventralsegmente fein punktiert, mit einzelnen groben, haartragenden Punkten, Endrand blafs, gelblich gefranst. Beine schwarz, Tarsen braun, weifslich behaart, alle Tarsen braun behaart, Scopa aufsen bräunlich. Flügel getrübt, Adern und Tegulae braun. - L. $8 \mathrm{~mm}$, Br. $3 \mathrm{~mm}$.

$\sigma^{7} \operatorname{der} A$. riparia sehr ähnlich, aber grölser und besonders durch die gekerbte Antenne zu unterscheiden. Abdomen schwach gelbbraun befilzt, $1-5$ mit weifslicher Haarbinde am Rande, 
7 zweispitzig, Tarsen gelbbraun, alle stark verlängert. L. $7^{1 / 2} \mathrm{~mm}, \mathrm{Br} .2^{1 / 2} \mathrm{~mm}$.

$\sigma^{7}+$ zahlreich von Para erhalten, wo sie von A d olf D u cke im Dezember und Januar gefangen wurden.

$$
\text { Ancyloscelis rufogrisea n. sp. ơ ㅇ. }
$$

Durch die eigenartig graue Behaarung $\left(\sigma^{7} q\right)$ des Kopfes und Thorax wie die rotgelbe des Abdomens auffallend.

․ Schwarz, grau behaart, auf Scheitel und Scutellum auch mit einzelnen schwarzen Haaren, Kopf und Thorax ziemlich grob runzlig-punktiert, die einzelnen Runzeln wieder äufserst fein gerunzelt, etwas glänzend, Kopf viel breiter $(1 / 2)$ als lang; Clypeus gewölbt, mit gelbem Endrand, Mandibelende rotgelb, Antenne schwarz, unten braun, zweites Geifselglied $=3+4$, Area des Mittelsegments glatt und glänzend. Abdomen fein und ziemlich dicht punktiert, Segment 1 mit fast weifslicher Basis, 2 mit gelbgrau befilzter Basis, Endrand von 2 und $3-5$ rotgelb befilzt, 5 mit viereckigem, schwarzem Haarfleck mitten am Rande, 6 schwarz, Analplatte querriefig. Ventralsegmente punktiert, gelbbraun gefranst, $5-6$ dicht braun befranst. Beine schwarz, grau behaart, Scopa weifslich, Metatarsus innen schwarzbraun behaart, Calcar rotbraun. Flügel hyalin, Endrand schwach getrübt, Adern braun, Tegulae hellbraun, durchsichtig. - L. $10 \mathrm{~mm}$, Br. $3^{1 / 2} \mathrm{~mm}$.

$\sigma^{7}$ wie + , aber Gesicht ganz schwarz, weifs behaart, Mandibelende rot; Brust schwarz behaart, Segment 1-6 am Endrande mit ziemlich breiten, lockeren Haarbinden, 7 schwarz behaart. Ventralsegmente schwarz, 2-5 mit rotgelben Borsten am Endrande, deren Spitzen umgebogen sind. Beine schwarz, grau behaart, doch Femur und Tibie meist schwarz behaart, Trochanter von II und III bürstenartig und schwarz behaart, Calcar schwarz, mit rotem Ende, bei den Beinen III der innere Calcar von doppelter Länge und Stärke. - L. $10 \mathrm{~mm}$, Br. $3 \frac{1}{2} \mathrm{~mm}$.

ơ $q$ mehrfach von Villa Rica (Paraguay), B u rg d o r f f leg.

$$
\text { Ancyloscelis latipes n. sp. or. }
$$

Der A. australis Cr. (Diadasia) ähnlich, aber gelbbraun behaart, aber Beine II kolossal verbreitert und Trochanter III verlängert und bewimpert.

$\sigma^{7}$. Schwarz, kurz gelbbraun behaart, Kopf fein und fast runzlig-punktiert, Clypeus und Labrum wie Mandibelbasis gelb, Mandibel sonst einfach und schwarzbraun; Antenne dünn, erreichen das Scutellum, zweites Geifselglied $=2 / 3$ so lang wie 3 . Thorax einzeln und zerstreut punktiert, Mesonotum auf der 
Scheibe mit grofsen, glatten Zwischenräumen, etwas glänzend: Abdomen schwarz, stark eingekrümmt, wie bei Anthidium mit braunen Segmentbinden, Segment 1 lang behaart, 2-6 mit braunfilziger Endhälfte, 7 rotbraun befilzt, auch die gerundete, scharf gerandete Analplatte, Segment 6-7 seitlich je mit spitzem Dorn; Ventralsegmente sehr stark und lang behaart, 6 konkav mit gescheitelten Haaren und kahler Mitte. Beine schwarz, Tarsen braun, Beine II stark deformiert, Trochanter verlängert, stumpf zweiteilig, Femur verbreitert, dreikantig, Tibie wie das Tarsenglied 1 tellerartig verbreitert, flach und fast von gleicher Gröfse, lang behaart, Trochanter III wie II verlängert und in eine scharfe, ganzrandige Kante endend, die mit einzelnen $(6-8)$ steifen, gekrümmten Borsten besetzt ist, Calcar gelb. Flügel gelblich getrübt mit dunklerem Rande, Adern braun, Tegulae schwarzbraun und behaart. - L. 11-12 mm, Br. $4 \mathrm{~mm}$.

$2 \sigma^{\top}$ von Villa Rica (Paraguay), B u rgdorff leg.

$$
\text { Ancyloscelis nigra n. sp. 중. }
$$

Durch die kurze und schwarze Behaarung auffallend, wie A. nigerrima, aber nur $1 / 2$ so grofs und dichter behaart.

․ Schwarz, kurz schwarz behaart, Kopf und Thorax fein punktiert, glänzend, Clypeus grober und körnig punktiert, Labrum gerundet, vor dem Endrand mit 2 kleinen Grübchen; Antenne kurz, keulenförmig, Geifselglied 2 fast so lang wie $3+4$. Mesonotum auf der Scheibe fast glatt, wie poliert glänzend, Area äufserst fein skulpturiert und dadurch gegen die Umgebung abstechend. Abdomen sehr fein punktiert, glänzend, dicht und kurz schwarz behaart, Analplatte schwarz, sehr schmal und parallel; Ventralsegmente mit braunen Endrändern, schwarz gefranst. Beine schwarz, Tarsen braun, schwarz behaart, Scopa rein schwarz, Calcar sehr lang und schwarz. Flügel hyalin, Adern schwarzbraun, Tegulae schwarz, glatt. - L. $7 \frac{1}{2}-8 \mathrm{~mm}$, Br. $3 \mathrm{~mm}$.

$\sigma^{7}$ wie $q$, schwarz behaart, Segment 7 zweiteilig, Ventralsegment 6 gerundet. Beine lang schwarz behaart, Tarsen verlängert. - L. $7 \mathrm{~mm}, \mathrm{Br} .3 \mathrm{~mm}$.

2 † $1 \sigma^{7}$ von Mendoza in $1200 \mathrm{~m}$ Höhe, im November 1908 von P. Jörge ns e n beobachtet.

Tetralonia spiniventris n. sp. $\sigma^{7}$.

?1909. T. flaviventris Jörgensen, $\sigma^{7}$, in: Deutsch. Ent. Zeitschr. p. 62.

Der T. jenseni Friese o äufserlich sehr ähnlich, aber Antenne ganz schwarz, sehr dick und viel länger, erreichen das zweite Segment, mit gebogenen Gliedern, so dafs sie gekerbt oder gesägt erscheinen. 
$0^{7}$. Schwarz, ganz dicht gelbbraun behaart, wie T. jenseni, aber Antenne länger, dicker und gekerbt, zweites Geifselglied sehr kurz, fast wie das erste. Thorax grob runzlig-punktiert, aber doch glänzend. Abdomen mit rotgefärbten Segmenträndern, aber dicht gelblich befilzt; Bauch kahl, Segment 6 mit langem, spitzem Dorn auf der Scheibenmitte bewehrt (sekundäres Kopulationsorgan!). Beine verdickt und verlängert, Tibie III etwas verbreitert und innen abgeplattet, glatt und unbehaart, Tarsen alle rotgelb, Metatarsus gekrümmt, Tarsenglied der Beine II etwas verbreitert und stark behaart. Flügel hyalin, Tegulae gelblich, Adern braun. - L. $10 \mathrm{~mm}$, Br. $3^{1} / 2 \mathrm{~mm}$.

4 ơ von Mendoza (J ö r g e n s e n leg.).

T. spiniventris kann identisch mit der flaviventris Jörgensen sein, doch stimmen einige wichtige morphologische Bildungen in den Beschreibungen nicht überein und bei den zahlreichen, gleichgefärbten und gleichgrofsen Arten sind die morphologischen Bildungen besonders zu betonen.

\section{Lasiocala Arrowi Ohs. (Col.)}

Von Dr. Fr. Ohaus, Steglitz-Berlin.

Von dieser Art, die ich kürzlich in den Ann. Belg. 1910 p. 221 beschrieb, kenne ich nun dank der Liebenswürdigkeit von Herrn Prof. Helle r in Dresden auch das + , das in folgenden Punkten vom $\sigma^{7}$ abweicht. Das fünfte Glied der Vordertarsen ist verdickt, die innere Klaue an der Basis breit, auffallend kurz, kürzer als die äufsere, die leicht hakenartig gekrümmt ist. An den Mittel- und Hinterfüfsen sind die Tarsen kürzer als beim $\sigma^{7}$, beide Klauen einfach und nahezu gleich lang, die äufsere etwas kürzer als die innere, durch das mit 2 Borsten besetzte Omychium soweit auseinander gedrängt, dals sie, wie bei vielen Melolonthiden, breit klaffen. Die Afterdecke ist undeutlich der Länge nach gefurcht, das mittlere und basale Drittel runzlig matt, das apicale dagegen glänzend mit vereinzelten Borstenpunkten. Die Fühler sind neungliedrig, indem das vierte und fünfte Geifselglied des $\sigma^{7}$ hier zu einem verschmelzen, die dreigliedrige Keule kürzer als die Geifsel. 


\section{$2 \mathrm{BHL}$ Biodiversity Heritage Library}

Friese, Heinrich. 1910. "Neue Bienenarten aus Süd-Amerika." Deutsche entomologische Zeitschrift 1910(6), 693-711. https://doi.org/10.1002/mmnd.4801910609.

View This Item Online: https://www.biodiversitylibrary.org/item/110145

DOI: https://doi.org/10.1002/mmnd.4801910609

Permalink: https://www.biodiversitylibrary.org/partpdf/236037

\section{Holding Institution}

California Academy of Sciences

\section{Sponsored by}

California Academy of Sciences Library

\section{Copyright \& Reuse}

Copyright Status: Public domain. The BHL considers that this work is no longer under copyright protection.

This document was created from content at the Biodiversity Heritage Library, the world's largest open access digital library for biodiversity literature and archives. Visit BHL at https://www.biodiversitylibrary.org. 